\title{
Current acceleration from the dilaton and stringy cold dark matter
}

\author{
Tirthabir Biswas, ${ }^{1}$ Robert Brandenberger, ${ }^{1}$ Anupam Mazumdar, ${ }^{2}$ and Tuomas Multamäki ${ }^{2}$ \\ ${ }^{1}$ Physics Department, McGill University, Montreal, Quebéc H3A 2T8, Canada \\ ${ }^{2}$ NORDITA, Copenhagen, Blegdamsvej-17, DK-2100, Denmark
}

(Received 20 October 2005; published 5 September 2006)

\begin{abstract}
We argue that string theory has ingredients to provide us with candidates for cold dark matter and explain the current acceleration of our universe. In any generic string compactification the dilaton plays an important role, as it couples to the standard model and other heavy nonrelativistic degrees of freedom such as string winding modes and wrapped branes, which we collectively call stringy cold dark matter. These couplings are nonuniversal, which results in an interesting dynamics for a rolling dilaton. Initially, it can track radiation and matter while beginning to dominate the dynamics recently, triggering a phase of acceleration. This scenario can be realized as long as the dilaton also couples strongly to some heavy modes. We furnish examples of such modes. We provide analytical and numerical results and compare them with the current supernovae result. We also mention some of the challenges for the success of our model favoring certain stringy candidates.
\end{abstract}

DOI: 10.1103/PhysRevD.74.063501

PACS numbers: $98.80 . \mathrm{Cq}$

\section{INTRODUCTION AND SUMMARY}

If string theory is the right paradigm for quantum gravity, then it must provide us with all the ingredients to make the currently observed universe. According to the most recent measurements [1], the universe is, to a good approximation, spatially flat, and the current energy budget of the universe consists of about 30\% nonrelativistic matter, most of it being weakly interacting and known as cold dark matter (CDM), and about $70 \%$ is in the form of an unknown source of dark energy which is responsible for the current acceleration. Unless a dominant fraction of them is hidden in dark astrophysical objects, standard model (SM) baryons can only make up a small fraction of the total energy density.

Obtaining the SM sector from string theory has been a hot pursuit; for a review, see [2]. However, in making the present universe, it is equally challenging to seek a candidate for cold dark matter which cannot be otherwise explained within the SM [3]. Finally and most importantly, one also has to obtain the vacuum/dark energy which seems responsible for the current acceleration $[1,5]$.

Now it is well known that any string and/or supergravity compactification to four space-time dimensions usually $[6,7]$ leads to a runaway potential for the dilaton (see [8] for efforts to stabilize the dilaton). Let us study the rolling dilaton dynamics in light of the dilaton's coupling to various matter fields. It is important to realize that the dilaton does not have a universal coupling to all the matter degrees of freedom [9], and it is this fact that we are going to exploit in our paper [10]. In order to have a holistic cosmological evolution in our model, we would require the following ingredients:

(1) The CDM candidate should be treated on an equal footing as the SM baryons in order to explain why $\Omega_{B} / \Omega_{\mathrm{CDM}} \sim \mathcal{O}(1)$. Therefore, if we assume that the dilaton couples to both the sectors, then it should have a similar coupling. In summary, both the SM and the CDM components should couple weakly (or be uncoupled) to the dilaton, in order to comply with various empirical constraints from fifth force experiments, from observational bounds on the variation of the fine structure constant and of Newton's constant, etc.

(2) In order to explain the current acceleration, we further require another CDM sector which is strongly coupled to the dilaton, and which we coin SCDM. We will see that, by virtue of its coupling to the dilaton, SCDM redshifts much more slowly than $\mathrm{CDM}$ and therefore may dominate the matter energy density at late times even if it was subdominant at early times. Moreover, if the coupling is strong enough, it slows down the rolling of the dilaton, causing the energy-momentum tensor of the dilaton to lead to an accelerated expansion of the universe.

We will provide examples of both standard CDM and SCDM in the context of string theory. Introducing a new form of dark matter may look ad hoc at first glance, but as we shall see such states are already present in string theory.

Moreover, our approach to explaining dark energy has an obvious advantage: no new extremely light, $m \sim$ $10^{-33} \mathrm{eV}$, scalar field is required to explain the origin of dark energy unlike in quintessence models [11-13] (for alternative explanations, see [14]). The natural scale in our approach is solely governed by the string scale. In this respect our model is minimal and facilitates making connections to string theory. The reason why acceleration starts at a much lower scale than the Planck scale, as we argue, can be traced back to having small initial cosmological energy densities of SCDM string states in the early (post-inflationary) universe.

We begin our discussion with the string theoretic motivation for CDM and SCDM (Sec. II). We explain how the 
string and brane degrees of freedom we make use of (which are the same ones studied in brane-gas cosmology $[15,16]$; see also [17]) couple to the dilaton, and give examples of candidates for SCDM which couple to the dilaton in a way which can give rise to acceleration. In Secs. III and IV, we provide analytical and numerical descriptions, respectively, of the evolution of the four distinct components in our cosmology, namely, radiation, CDM, SCDM, and the dilaton. We also constrain the couplings by fitting to the supernovae data. In Sec. V, we readdress the cosmic acceleration problem within the string theoretic framework. We conclude by summarizing and pointing to future research challenges.

\section{MOTIVATION FROM STRING THEORY}

\section{A. Dilaton gravity}

The main aim of this section will be to find two important couplings, first the dilaton self-coupling which will determine the slope of the runaway potential, and second the coupling of the dilaton to the stringy degrees of freedom, allowing us to identify stringy candidates for CDM and SCDM.

Let us start with 10-dimensional type II string theory whose low energy bosonic action is given by

$$
\begin{aligned}
\hat{S}_{I I}= & \frac{1}{16 \pi \hat{G}} \int d^{10} x \sqrt{-g}\left[e ^ { - 2 \phi } \left(\hat{R}+4 \partial_{\hat{m}} \phi \partial^{\hat{m}} \phi-V(\phi)\right.\right. \\
& \left.\left.-\frac{1}{12} H_{3}^{2}\right)-\sum_{n} e^{a_{n} \phi} \frac{1}{2 n !} F_{n}^{2}\right]
\end{aligned}
$$

where hatted quantities denote the full higher $(\hat{D}=10)$ dimensional objects, $\phi$ is the dilaton, $H_{3}$ is the field strength of the antisymmetric tensor field, and the field strengths $F_{n}$ 's are $n$ forms (where $n=2,4$ in type IIA theory while $n=1,3,5$ in type IIB theory). To be general, we have also added a potential for the dilaton. Such a potential could result from quantum corrections $[6,7]$.

We will assume that all shape and volume moduli of the internal manifold are stabilized. This could occur by making use of the GKP scenario [19] to stabilize the complex structure (shape) moduli with the help of various form fields, and then invoking the KKLT ideas [20] to stabilize the volume modulus with the help of nonperturbative processes. Alternatively, but only in heterotic string theory, we could use ideas from brane-gas cosmology $[15,16]$ to stabilize both volume [21-23] and shape [24] moduli by means of string states which are massless at the self-dual radius (see also [25] for a study of volume stabilization in the string frame which is applicable both to type II and heterotic string theories). We assume that these moduli do not play any substantial role in the late-time dynamics (this assumption is supported by the analyses of $[22,23]$ ), and henceforth we will ignore them.

Performing the well-known conformal transformation (relating the string-frame metric on the left-hand side to a function of the dilaton multiplying the Einstein frame metric on the right-hand side)

$$
\hat{g}_{\hat{m} \hat{n}} \rightarrow e^{4 \phi /\left(\stackrel{D}{D}^{2}\right)} \hat{g}_{\hat{m} \hat{n}},
$$

(where $\stackrel{\circ}{D}$ is the number of compactified spatial dimensions), we recover from (1) an action in the 10-dimensional Einstein frame,

$$
\hat{S}=\frac{1}{16 \pi \hat{G}} \int d^{10} x \sqrt{-\hat{g}}\left\{\hat{R}-\frac{1}{2} \partial_{\hat{m}} \phi \partial^{\hat{m}} \phi-2 V(\phi)\right\},
$$

where the new potential is related to the original one by rescaling:

$$
V(\phi) \rightarrow e^{\phi / 2} V(\phi) .
$$

We take the full higher-dimensional metric to be

$$
\hat{g}_{\hat{m} \hat{n}}=\left(\begin{array}{cc}
g_{m n}(x) & 0 \\
0 & r^{2} g_{m i}^{\circ}(y)
\end{array}\right),
$$

where $r$ is the radius (volume) modulus of the compact space and where we used the symbol "o" to indicate extradimensional quantities.

Then, after integrating over the extra dimensions, one straightforwardly obtains

$$
S=\frac{M_{p}^{2}}{2} \int d^{4} x \sqrt{-g}\left[R-\partial_{m} \phi \partial^{m} \phi-2 V(\phi)\right],
$$

where we have defined

$$
M_{p}^{2}=M_{s}^{8} r^{6}
$$

as the four space-time-dimensional Planck mass, and we have also rescaled $\phi$,

$$
\phi \rightarrow \sqrt{\frac{1}{2}} \phi,
$$

so that it has a canonical kinetic term.

As in [26], we now specialize to the case when $V(\phi)$ is an exponential potential,

$$
V(\phi)=V_{0} e^{-2 \beta \phi} .
$$

Indeed, such exponential potentials are common in lowerdimensional supergravity models and can also arise in string theory from quantum corrections (both perturbatively, for example, involving string loops [6,9], or nonperturbatively, like in gaugino condensation [7]). Although we keep $\beta$ as a free parameter in our model, ideally one should be able to derive its value from the origin of the potential, and we discuss an example in Sec. V.

\section{B. Wrapped branes as candidates for CDM and SCDM}

Branes and strings can wrap certain cycles in the internal dimensions. In general, they are heavy objects with mass scales of the order of the string scale (exceptions are the 
special modes which are massless at the self-dual radius however, such modes exist in heterotic theory but not in type II string theory). The dilaton, in general, couples to these objects, and here we determine these couplings. As in the usual brane-gas cosmology paradigm $[15,16]$, we assume that branes have annihilated in the three noncompact directions, allowing them to expand, and leaving us only with branes wrapping some or all of the six internal extra dimensions. Hence we only need to compute the couplings for $p$-branes with $p=2, \ldots, 6$, and for the string winding and momentum modes.

The brane couplings can be derived [27,28] starting from the Dirac-Born-Infeld (DBI) action, which in the string frame reads

$$
S_{\mathrm{DBI}}=M_{s}^{p+1} \int d^{p+1} \sigma e^{-\phi} \sqrt{-\gamma},
$$

where $\gamma$ is the metric on the brane world sheet labeled by $\sigma$. One has to now make conformal transformations (2) followed by dilaton rescaling (8) to obtain the brane coupling $\mu_{p}$ in the Einstein frame:

$$
S_{\mathrm{DBI}}=M_{s}^{p+1} \int d^{p+1} \sigma e^{2 \mu_{p} \phi} \sqrt{-\gamma}
$$

with

$$
2 \mu_{p}=\frac{p-3}{\sqrt{8}} .
$$

The wrapped branes of course appear to us, from the large four space-time-dimensional point of view, as pointlike objects. Thus, their number density redshifts as $a^{-3}$, like nonrelativistic dust. In the brane-gas approximation the four-dimensional action for wrapped $p$-branes is therefore given by (see e.g. [22])

$$
S_{\text {brane }}=\int d^{4} x \sqrt{-g} \rho_{0} e^{2 \mu_{p} \phi}\left(\frac{a}{a_{0}}\right)^{-3} \equiv \int d^{4} x \sqrt{-g} \tilde{\rho}_{p},
$$

where $\tilde{\rho}_{p}$ corresponds to the observed four-dimensional energy densities of the wrapped branes which appear in the Einstein equations [29]. Note that, because of the coupling, if $\mu_{p}$ is positive the energy density $\tilde{\rho}_{p}$ redshifts slower than $a^{-3}$ if the dilaton increases, and this is why these wrapped branes can act as candidates for SCDM.

By an analogous prescription we can also obtain the four-dimensional action for string winding modes [30], and momentum modes starting from the Polyakov action [31]. For the winding modes we find

$$
2 \mu_{1}=\frac{1}{\sqrt{2}}
$$

while the momentum modes do not couple to the dilaton at all [31]:

$$
\mu_{\text {mom }}=0
$$

One immediately realizes that, since the momentum string modes do not couple to the dilaton, their energy density redshifts in the same way as baryons, i.e. as $a^{-3}$, and therefore they can be candidates for CDM. On the other hand, the coupled winding states can form SCDM. However, since we want SCDM to become important (comparable to CDM) only at the present cosmological epoch, this means that in the early universe the energy density of the winding states must have been much smaller than that of the momentum modes. Here, we present a qualitative argument based on the masses of these objects as to why we expect this to be the case. We will address the issue more quantitatively in Sec. V B.

From Eq. (12) we can directly read off the masses of the $p$-branes as

$$
m_{p} \sim M_{s}^{p+1} r^{p},
$$

where $r$ is the radion field in the Einstein frame. We assume that the radion is stabilized. Similarly, from the Polyakov action the masses for the string winding and momentum modes are given by

$$
m_{1} \sim M_{s}^{2} r
$$

and

$$
m_{\mathrm{mom}} \sim \frac{1}{r} .
$$

For the purpose of illustration, here we have assumed that, initially, $\phi$ evolves from close to zero, but similar arguments can be made for more general initial conditions.

If the radion is stabilized at a scale slightly lower than the Planck scale, say $r^{-1} \sim M_{\mathrm{GUT}}$, then, because of the $r$ dependence of the different masses, the momentum modes turn out to be the lightest, while all the winding string modes and branes are much heavier. Thus, if there was a period of inflation of the three large spatial dimensions, and if the temperature after reheating is of the GUT scale, then the reheating process at the end of inflation can either perturbatively $[32,33]$ or via parametric resonance $[34,35]$ produce string momentum modes [36]. The thermal production of the winding states (which are very heavy compared to the scale of inflation) is exponentially suppressed. There are, however, some mechanisms by which such heavy modes could be produced [38,39], although their number densities would be much smaller than that of the momentum modes. Thus, in the context of inflationary cosmology, this may yield one way to explain the difference in the initial post-inflationary energy densities of the momentum string modes (CDM) and the winding modes (SCDMs). It is possible, however, that the winding mode production processes after inflation are too weak. In this case, one must invoke the primordial preinflationary density of SCDM states to determine the late-time number density: in Sec. V we argue how a period of inflation of appropriate length can dilute primordial energy density of 
SCDMs to make it just right to address the coincidence problem.

If both baryons and CDM momentum states are produced during reheating after inflation, then it should be expected that their densities are comparable, as long as the masses of both types of states are small compared to the typical energy scale during reheating. If the initial postinflationary baryon density is not changed substantially by later stages of baryon production, the number densities of baryons and CDM particles are expected to be comparable.

Let us then assume that after inflation we have very small energy densities of the different wrapped brane/ string gases. Now observe that, because of the difference in the coupling exponents $\mu_{p}$ 's, all the species redshift differently. As $\phi$ is increasing, it is clear that very soon the largest energy density among these different species would correspond to the one with the largest positive $\mu_{p}$. From (12) and (14), one finds that this corresponds to the 6branes, with

$$
\mu_{6}=\frac{3}{2 \sqrt{8}} .
$$

Thus, according to our scenario, at late times the dark sector of our universe will consist of three main components: (a) the dilaton behaving as dark energy, (b) string momentum modes playing the role of ordinary CDM, and (c) 6-branes, which wrap the six extra dimensions, playing the role of SCDM. As we shall see in the next section, by virtue of their coupling (19) to the dilaton, 6-branes can trigger a late phase of acceleration.

\section{COSMOLOGICAL MODEL}

\section{A. Acceleration due to coupling: basic mechanism}

We begin by analyzing a simple scalar-tensor theory action for dilaton gravity, where the dilaton has a runaway potential:

$$
S_{\text {grav }+\phi}=M_{p}^{2} \int d^{4} x \sqrt{-g}\left[\frac{R}{2}-\frac{(\partial \phi)^{2}}{2}-V_{0} e^{-2 \beta \phi}\right] .
$$

In principle, $\phi$ can be identified with any suitable moduli field, as exponential potentials are quite common for other kinds of moduli fields as well, for example, in flux compactifications [40]. However, for the purpose of this paper we focus on the dilaton. As in conventional cosmology, gravity is sourced by the matter-radiation stress energy tensor which can be derived from an action of the form

$$
S_{i}=\int d^{4} x \sqrt{-g} \tilde{\rho}_{i}
$$

where the index $i$ represents the different components of matter/radiation and $\tilde{\rho}_{i}$ is the observed energy density that gravitates. The Hubble equation reads

$$
H^{2}=\frac{1}{3}\left(\sum_{i} \tilde{\rho}_{i}+\frac{1}{2} \dot{\phi}^{2}+V(\phi)\right) \equiv \frac{1}{3}\left(\sum_{i} \tilde{\rho}_{i}+\tilde{\rho}_{\phi}\right) .
$$

Note there is a departure from the conventional cosmology. We allow different matter components to couple to $\phi$ :

$$
\tilde{\rho}_{i}=e^{2 \mu_{i} \phi} \rho_{i},
$$

where we further assume that the $\phi$-independent "bare density" $\rho_{i}$ behaves like a perfect fluid, i.e., it obeys the continuity equation:

$$
\dot{\rho}_{i}+3 H\left(p_{i}+\rho_{i}\right)=0,
$$

with an equation of state $p_{i}=\omega_{i} \rho_{i}$.

If a scalar field $\phi$ is to act as a quintessence type field, then it must be virtually massless, i.e. $m_{\phi} \sim 10^{-33} \mathrm{eV}$. If $\phi$ is coupled to SM particles, it mediates a fifth force. Fifth force experiments constrain such couplings of $\phi$ to SM degrees of freedom to be very small, $\mu_{\mathrm{SM}}<10^{-4}-10^{-5}$ (see e.g. [41]). Observations on variation of physical constants like the fine structure and Newton's constants also give us similar bounds on $\mu_{\mathrm{SM}}$. To be able to explain such small couplings is, in fact, a generic and challenging problem in quintessence models. However, one finds that in our specific scenario where the dilaton evolves to a strong coupling regime $(\phi \gg 1)$ such small couplings may have a natural explanation [42], as discussed in [6]: In this setup one expects the masses, $m_{b}(\phi)$ 's, and gauge couplings, $g(\phi)$ 's, for standard model particles to be given by a Taylor series expansion in inverse string coupling $\left(g_{s}^{-1} \sim e^{-\phi}\right)$ [6]. For example, if $\alpha_{\mathrm{GUT}}$ is the GUT-scale fine structure constant, then we have

$$
\alpha_{\mathrm{GUT}}=\alpha_{0}+\alpha_{1} e^{-\phi}+\cdots .
$$

Now, the crucial thing to note is that observations really only constrain the dimensionless ratios

$$
\frac{1}{m_{b}} \frac{d m_{b}}{d \phi}, \frac{1}{g} \frac{d g}{g d \phi}<10^{-4}-10^{-5} .
$$

For large values of $\phi$, one can easily estimate these quantities. For instance, from (25)

$$
\frac{1}{\alpha_{\mathrm{GUT}}} \frac{d \alpha_{\mathrm{GUT}}}{d \phi} \sim \frac{\alpha_{1} e^{-\phi}}{\alpha_{0}} \sim e^{-\phi} .
$$

Thus, as long as the constant term in the expansion is nonzero (in general, it is nonzero, and is determined by the various group theoretical quantities of the gauge group [6]) the ratio becomes smaller and smaller as $\phi$ evolves to larger values and one can easily satisfy the observational bounds.

At this point one may worry about other stringy corrections that may be present in the effective action: for instance, there could be $\alpha^{\prime}$ corrections but these are expected to be small since they are suppressed by the string scale and typically we are dealing with much smaller energies. On the other hand, one could have string loop corrections, 
which do not necessarily conform to the perturbative expansions such as (25), potentially invalidating our argument. However, a detailed study of these effects is well beyond the scope of our paper and we will assume that (25) is valid, at least in some parameter range. We have discussed this issue in the Appendix.

Therefore, in the following we will assume that radiation $(r)$, baryons $(b)$, and CDM (string momentum modes) have tiny/no couplings to $\phi$.

The dynamics of $\phi$ depends on the exponent $\beta$, and the coupling of $\phi$ to various matter-radiation components. The equation of motion for $\phi$ is given by

$$
\ddot{\phi}+3 H \dot{\phi}=-V_{\text {eff }}^{\prime}(\phi),
$$

with

$$
V_{\mathrm{eff}}(\phi)=V_{0} e^{-2 \beta \phi}+\sum_{i=m, \mathrm{sc}} e^{2 \mu_{i} \phi} \rho_{i},
$$

where the sum runs over all matter states. For simplicity, we have assumed that the gauge bosons do not contribute to an effective potential for $\phi$. This is strictly true if, on average, the electric and magnetic fields vanish. There could be other forms of radiative matter which could induce an effective coupling, as discussed, for example, in [44]. Weakly coupled matter (stringy candidates for CDM and baryons) are denoted by the subscript " $m$," and SCDM by the subscript "sc."

It was argued in [26] that, when the universe evolves adiabatically, the dilaton $\phi$ tracks the minimum of the effective potential which evolves in time with the redshifting of the $\rho_{i}$ 's. How fast or slow the dilaton is evolving mostly depends on the equation of state parameter $\omega_{i}$, and on the coupling exponent $\mu_{i}$ of the dominating matter component. Thus, at earlier times when radiation or weakly coupled matter is dominant, $\phi$ rolls fast and tracks the relevant matter/radiation component, remaining subdominant. However, once SCDM becomes important, the dilaton slows down, starts to dominate the energy density, and our universe enters a phase of acceleration. Let us now see this in more detail.

\section{B. Exact two-fluid analysis}

We will first work within the approximation that there are only two dominating components in the universe, the dark energy determined by $\phi$ and a single matter/radiative component. To keep things general, we do not specify which form of matter it is. In this approximation, the Einstein constraint equation (22) determining the Hubble expansion rate reads

$$
H^{2}=\frac{1}{3}\left(\mathrm{e}^{2 \mu \phi} \rho+\frac{1}{2} \dot{\phi}^{2}+V(\phi)\right) .
$$

From Eq. (27), it follows that the evolution equation for $\phi$ is given by

$$
\ddot{\phi}+3 H \dot{\phi}=2\left(\beta V_{0} \mathrm{e}^{-2 \beta \phi}-\mu \mathrm{e}^{2 \mu \phi} \rho\right) .
$$

Solving the continuity equation (24), we obtain

$$
\rho=\rho_{0}\left(\frac{a}{a_{0}}\right)^{-3(1+\omega)}
$$

where $\rho_{0}$ is the present-day value of the energy density.

Performing a change of variables $\eta=\ln (a(t))$ leaves us with the following set of equations:

$$
\begin{aligned}
H^{2} & =\frac{\frac{1}{3} \mathrm{e}^{2 \mu \phi} \rho+V_{0} \mathrm{e}^{-2 \beta \phi}}{1-\frac{1}{6} \phi^{\prime 2}}, \\
\phi^{\prime \prime}+\left(3+\frac{H^{\prime}}{H}\right) \phi^{\prime} & =2 \beta \frac{V_{0}}{H^{2}} \mathrm{e}^{-2 \beta \phi}-2 \mu \frac{\rho}{H^{2}} \mathrm{e}^{2 \mu \phi},
\end{aligned}
$$

where ${ }^{\prime} \equiv d / d \eta$. The system of equations can be solved analytically to obtain the "tracking solution," the solution where $\phi(t)$ tracks the minimum of its "effective" potential. The solution is given by

$$
e^{\phi}=\left[\frac{V_{0}}{\rho}\left(\frac{\beta(\beta+\mu)-3 / 4(1+\omega)}{\mu(\mu+\beta)+3 / 4(1+\omega)^{2}-3 / 8(1+\omega)}\right)\right]^{p}
$$

where

$$
p=\frac{1}{2(\beta+\mu)}
$$

and

$$
a(t)=a_{0}\left(\frac{t}{t_{0}}\right)^{2 /\left[3\left(1+\omega_{\phi}\right)\right]} \quad \text { with } \omega_{\phi}=-\frac{\mu / \beta-\omega}{\mu / \beta+1} .
$$

A few remarks are now in order. First, we observe that the energy densities $\tilde{\rho}$ and $\tilde{\rho}_{\phi}$ track each other and redshift with precisely the same equation of state parameter $\omega_{\phi}$ that was derived in the adiabatic approximation in [26]:

$$
\begin{aligned}
\tilde{\rho}_{\phi} & =\frac{\mu(\mu+\beta)+3 / 4(1+\omega)^{2}-3 / 8(1+\omega)}{\beta(\beta+\mu)-3 / 4(1+\omega)} \tilde{\rho} \\
& \equiv r \tilde{\rho} \sim a^{-3\left(1+\omega_{\phi}\right)} .
\end{aligned}
$$

One recovers the value of the tracking ratio

$$
r=\frac{\mu}{\beta},
$$

obtained in the adiabatic approximation [26] when $\mu, \beta \geq$ $\mathcal{O}(1)$ and the terms involving $(1+\omega)$ can be ignored.

Second, we realize that real solutions for $\phi$ only exist if the numerator on the right-hand side of (32) is positive (the denominator is positive as long as $\omega>-1 / 2$ ). In other words, in order for a tracking solution to exist, we require

$$
\beta(\beta+\mu)>\frac{3(1+\omega)}{4} .
$$

Since we want such tracking behavior to hold during the radiation-dominated phase, substituting $\mu_{r}=0$ and $\omega_{r}=$ $1 / 3$ in Eq. (37), we find 


$$
\beta>1 .
$$

We note in passing that, if the condition Eq. (37) is not satisfied, then we end up with a different, nontracking, latetime attractor solution. In this case the field does not follow the minimum but lags more and more behind as the universe expands, and the energy density becomes dominated by the dilaton from the very beginning. From the point of view of cosmology, this is an uninteresting scenario.

Finally, we find from Eqs. (34) that acceleration happens when

$$
\frac{\mu_{\mathrm{sc}}}{\beta}>\frac{3}{2}\left(\frac{1}{3}+\omega_{\mathrm{sc}}\right) .
$$

For winding brane $\operatorname{SCDM}\left(\omega_{\mathrm{sc}}=0\right)$, combining (39) with (38), we find that we can account for a late acceleration phase provided our SCDM has a coupling exponent,

$$
\mu_{\mathrm{sc}}>\frac{1}{2} \text {. }
$$

Note, in particular, that the exponent which we computed for wrapped 6-branes satisfies this bound.

\section{Three-fluid system}

Our exact analysis of the two-fluid model suggests that, if the dilaton couples to SCDM, then there can be an interesting dynamics which triggers a phase of acceleration. In order to study more precisely how such a transition happens, one has to consider a more realistic cosmological model where, in addition to the SCDM fluid, we also have a normal CDM component that is uncoupled or weakly coupled to the dilaton. Such a model has the advantage that, at early times, the evolution is standard, i.e. $H \sim$ $a^{-3 / 2}$, and, at late times, as SCDM plus dark energy begin to dominate, we can have accelerated expansion.

The new system of equations (22)-(27) becomes

$$
\begin{gathered}
H^{2}=\frac{1}{3 M_{p}^{2}}\left(\tilde{\rho}_{\phi}+\tilde{\rho}_{\mathrm{sc}}+\tilde{\rho}_{m}\right), \\
\ddot{\phi}+3 H \dot{\phi}=2\left(\beta V_{0} e^{-2 \phi}-\mu_{\mathrm{sc}} \rho_{\mathrm{sc}} e^{2 \mu_{\mathrm{sc}} \phi}-\mu_{m} \rho_{m} e^{2 \mu_{m} \phi}\right) \\
=2\left[\beta V(\phi)-\mu_{\mathrm{sc}} \tilde{\rho}_{\mathrm{sc}}-\mu_{m} \tilde{\rho}_{m}\right] .
\end{gathered}
$$

For simplicity, we assume for the time being that $\mu_{m}=0$; we will see later that, as long as $\mu_{m} \ll \beta$, it hardly makes any difference to our analysis. Although no analytical solution to Eqs. (41) and (42) is found, one can obtain some insight into the cosmological evolution resulting from these equations by considering various limiting situations.

First transition.-First, consider a situation when the energy density in SCDM is much smaller than that in both ordinary matter and in $V(\phi)$, so that one can neglect the second terms in Eqs. (41) and (42). In this case, the equations from the two-fluid discussion apply, making use of $\mu=0$ and $w=0$. This is the phase when the dilaton essentially rolls down freely along its potential $V(\phi)$, tracking the energy density of ordinary matter [26] as given by (35):

$$
\tilde{\rho}_{\phi}=\frac{3}{8 \beta^{2}-6} \tilde{\rho}_{m}
$$

The scale factor $a(t) \propto t^{2 / 3}$, as in the usual matterdominated era. Note that during this period the value of $\phi$ which is the minimum of the "effective potential" for $\phi$ [the sum of $V(\phi)$ and $\tilde{\rho}_{\text {sc }}$ ] is redshifting more slowly than the value of $\phi(t)$ in this phase [this can be seen by going back to (32), since it is setting $\mu=\mu_{\mathrm{sc}}$ in this equation which gives the time dependence of the minimum], and thus $\phi(t)$ eventually catches up with the minimum. After this point, the value of $\phi(t)$ tracks the minimum. In this case, we know that the energy density of $\phi$ will track SCDM, as given in Eq. (35). It is clear, then, that when the right-hand sides of Eqs. (35) and (43) become equal, i.e. when

$$
\frac{3}{4 \beta^{2}-3} \tilde{\rho}_{m}=\frac{\mu}{\beta} \tilde{\rho}_{\mathrm{sc}}
$$

and thus

$$
\tilde{\rho}_{\mathrm{sc}}=\frac{3 \beta}{\mu\left(4 \beta^{2}-3\right)} \tilde{\rho}_{m},
$$

a first transition, namely, the transition between the period when $\phi(t)$ is moving freely and when it starts tracking the minimum of the effective potential (set by the interaction of the dilaton potential and the contribution from the SCDM term), occurs [45]. From this moment on, both $\tilde{\rho}_{\text {sc }}$ and $\tilde{\rho}_{Q}$ start to redshift as Eq. (35), with $\omega \rightarrow 0$ and $\mu \rightarrow \mu_{\mathrm{sc}}$.

Second transition. - The second transition [46] corresponds to when the universe enters a phase of acceleration. This happens approximately when $\tilde{\rho}_{d} \equiv \tilde{\rho}_{\mathrm{sc}}+\tilde{\rho}_{\phi}=(1+$ $\left.r_{\mathrm{sc}}\right) \tilde{\rho}_{\mathrm{sc}}$ becomes equal to the energy density of the ordinary matter components, as can be readily seen from the Hubble rate, Eq. (41). Thus, we enter a phase of acceleration when

$$
\tilde{\rho}_{\mathrm{sc}}=\frac{1}{1+r_{\mathrm{sc}}} \tilde{\rho}_{m} \approx \frac{1}{1+\mu_{\mathrm{sc}} / \beta} \tilde{\rho}_{m},
$$

where in the last step we again made use of the adiabatic approximation.

First, notice that, depending on how large the ratio $\mu_{\mathrm{sc}} / \beta$ is, one needs a very small fraction of SCDM compared to ordinary weakly coupled matter to initiate the phase of acceleration. Since SCDM behaves like nonrelativistic dust $\left(\omega_{\mathrm{sc}}=0\right)$ it would cluster around galaxies and contribute to the current dark matter abundance, thereby changing the baryon to dark matter ratio in recent times. Since there is reasonable agreement between this ratio as obtained from the cosmic microwave background (CMB) (which measures the primordial fluctuations at redshifts of around $z=1100$ ) and measurements of rich clusters of 
galaxies (at recent redshifts), it is important that this ratio does not change too much in our model. This is guaranteed if $\tilde{\rho}_{\mathrm{sc}}$ is only a small fraction of $\tilde{\rho}_{m}$. As pointed out in [26], this scenario of changing baryon to dark matter abundance offers the intriguing possibility of reconciling a $10 \%$ to $20 \%$ discrepancy in the estimates of baryonic abundances coming from $\mathrm{CMB}$ and big bang nucleosynthesis (BBN) measurements [47].

What if SCDM is a radiative fluid? - So far, we have assumed that SCDM is a form of dark matter (winding states) but is strongly coupled to the dilaton. What if it were to behave as a radiative fluid? For example, in [44] it was proposed that thermal fluctuations of a massless field with quartic interactions can act as a radiative fluid with precisely the kind of exponential coupling to the dilaton that we considered here. In this case, $\omega_{\mathrm{sc}}=1 / 3$, and we will observe it as a component of dark energy. This is because, after the first transition, both SCDM and $\phi$ obey the same equation of state $\omega_{\phi}$ as computed in Eq. (35), and cosmologically we cannot distinguish between the two. The total dark energy would then be given by $\tilde{\rho}_{d}$. Obviously, in this setup the ratio between dark matter and baryons remains constant and we do not encounter any constraints on the ratio $\mu_{\mathrm{sc}} / \beta$ on this basis. From (39) we find that in order to have acceleration the ratio just needs to satisfy

$$
\frac{\mu}{\beta}>1
$$

Acceleration epoch.-In either case, the main constraint comes from trying to match the observed equation of state, $\omega_{\phi}<-0.76$ [1,5]. From Eq. (34) we find such a bound implies

$$
\frac{\mu_{\mathrm{sc}}}{\beta}>3
$$

for SCDM and

$$
\frac{\mu_{\mathrm{sc}}}{\beta}>5
$$

for radiation. How to get such a large value of the ratio from string theory is discussed in the following section.

For these ratios of $\mu_{\mathrm{sc}} / \beta$, one can also estimate approximately the onset of the period of acceleration. It is the time when $\tilde{\rho}_{d} \approx \tilde{\rho}_{m}$. Now,

$$
1=\frac{\tilde{\rho}_{d, \mathrm{acc}}}{\tilde{\rho}_{m, \mathrm{acc}}}=\frac{\tilde{\rho}_{d 0}}{\tilde{\rho}_{m 0}}\left(\frac{a_{\mathrm{acc}}}{a_{0}}\right)^{-3 \omega_{Q}}=\frac{\Omega_{d 0}}{\Omega_{m 0}}\left(\frac{1}{1+z_{\mathrm{acc}}}\right)^{-3 \omega_{Q}} .
$$

Using the known dark matter to dark energy ratio, we find

$$
z_{\mathrm{acc}} \sim 0.5-1.0,
$$

which is in good agreement with the supernova data.
Finally, we note that most of our analyses of the transitions go through identically even if $\mu_{m}$ is nonzero but small. This is because, if $\mu_{m}$ is tiny, it changes the tracking ratio very little in the matter phase (43), which enters in the calculation for the epoch of the first transition. Also,

$$
\tilde{\rho}_{m} \sim a^{-3+\mathcal{O}\left(\mu_{m} / \beta\right)} .
$$

Thus, weakly coupled matter redshifts essentially the same way as in the standard cosmology, and therefore the second transition is also unaffected.

To summarize, we have three distinct cosmological eras depending on which terms are most dominant on the righthand side of the Hubble equation (41):

(1) The radiation era, when $\tilde{\rho}_{\mathrm{sc}}$ and $\tilde{\rho}_{m}$ are negligible while $\tilde{\rho}_{\phi}$ tracks $\tilde{\rho}_{r}$

(2) The matter era, when $\tilde{\rho}_{\text {sc }}$ and $\tilde{\rho}_{r}$ are negligible while $\tilde{\rho}_{\phi}$ tracks $\tilde{\rho}_{m}$

(3) An acceleration phase, where stringy components, viz. $\tilde{\rho}_{d}$, start to dominate over $\tilde{\rho}_{m}$.

As far as the parameters of our model are concerned, first we find that, in order to have a late-time acceleration phase, we need the ratio $\mu_{\mathrm{sc}} / \beta$ to satisfy conditions like Eq. (62). On the other hand, to have an earlier nonaccelerating phase $\mu_{r}, \mu_{m}$ has to be small which is consistent with constraints coming from fifth force experiments, and observations of variation of $G_{N}$ and $\alpha_{f}$ which lead to the condition $\mu_{\mathrm{SM}}<10^{-5}$. Finally, the requirement that $\phi$ should track radiation in the early universe tells us that $\beta>$ 1. In spite of these constraints, there is a huge parameter space where we seem to agree with the cosmological and particle physics observations, which is encouraging. Let us therefore study this model in more detail using numerical techniques and see whether the allowed parameter space can be made more precise.

\section{NUMERICAL WORK}

\section{A. Evolution and abundances}

In order to verify our analytical approximations and see the precise evolution of the system, we solve the three-fluid system numerically. We start deep in the matter-dominated era and choose initial conditions such that the field initially follows the approximate early-time solution. As an example, the evolution of the field for the set of parameter values $\beta=6, \mu=10, \Omega_{\tilde{m}}=0.27, \Omega_{\mathrm{m}}=0.01$, and $V_{0}$ fixed by requiring flatness is shown in Fig. 1. The plot depicts the field value as a function of time (measured in terms of the logarithm of the scale factor). The solid red curve represents the full numerically determined evolution. In the same figure we also show the analytical approximations given by Eqs. (41) and (42) in a universe that is radiation dominated, i.e. $\exp (\phi)=\left(8 \beta^{2} V_{0} / 3 \rho_{r}\right)^{1 / 2 \beta}$ (dotdashed purple curve), dominated by ordinary matter, i.e. $\exp (\phi)=\left(8 \beta^{2} V_{0} / 3 \tilde{\rho}_{m}\right)^{1 / 2 \beta}$ (long-dashed green curve), or described by the late-time solution (dashed blue curve) 


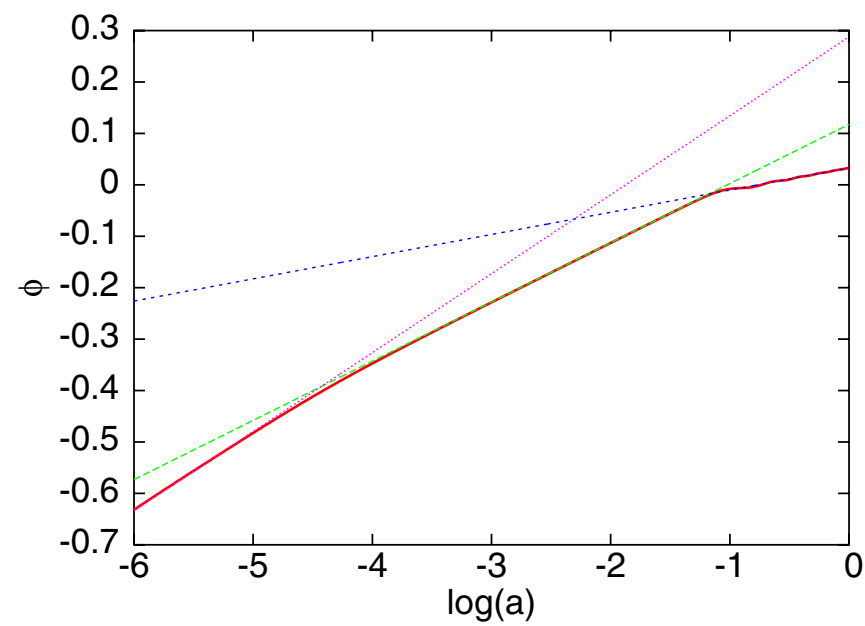

FIG. 1 (color online). The evolution of $\phi$ as a function of the logarithm of the cosmological scale factor for the full numerical solution (solid red line) and for the approximations to the solutions corresponding to the evolution at late (blue dashed line) times, early (green long-dashed line) times and in the initial radiation-dominated (purple dot-dashed line) phase.

when the field follows the effective minimum of the potential Eq. (35). From the figure, one can see how the field initially follows the radiation-dominated solution until it crosses the matter-dominated solution, after which the evolution of the fields is well described by it. At late times, the field follows the solution of the evolution of the minimum of the effective potential. Note also how the crossover from one solution to another is very rapid and smooth.

The relative contributions $\Omega_{i}$ of various matter constituents (labeled by $i$ ) to the critical density of a spatially flat universe are shown in Fig. 2. It is clear how at late times the

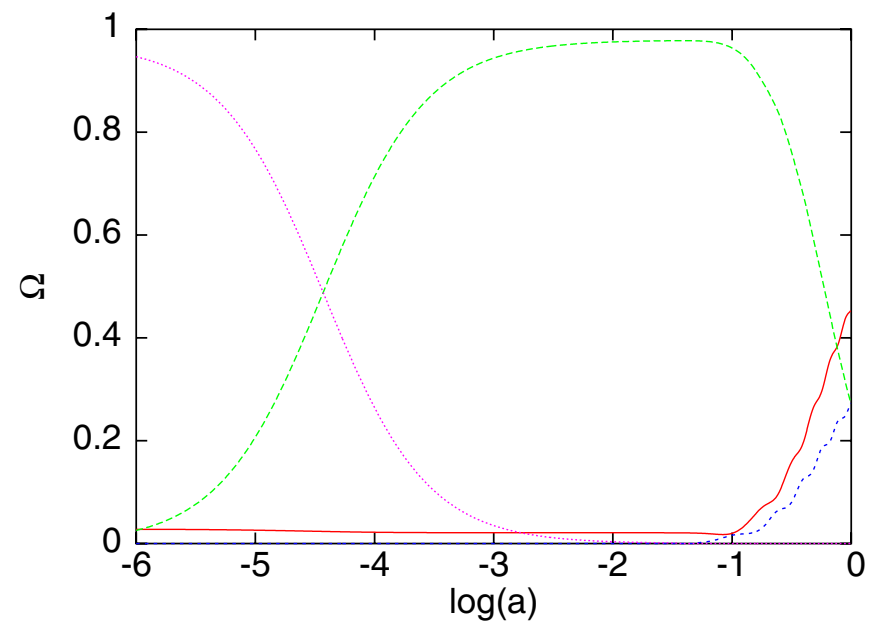

FIG. 2 (color online). The relative energy densities in the $\phi$ field (red solid line), in coupled CDM (SCDM) (blue dashed line), uncoupled CDM (green long-dashed line) and in radiation (purple dot-dashed line). coupled dark matter component starts dominating along with the $\phi$ field.

\section{B. Observational constraints: Supernova type Ia data}

In order to see whether the model presented here is compatible with present observational data, we compare it with the recent expansion history as probed by the supernova type Ia (SNIa) data. Here we use the sample of 194 SNIa presented in [48]. In order to compare our model with the $\Lambda \mathrm{CDM}$ model, we compute the $\chi^{2}$ for each set of parameters and compare it with the value for the corresponding flat $\Lambda \mathrm{CDM}$ value, which for the same set of supernovae is $\chi_{\Lambda \mathrm{CDM}}^{2}=201.6\left(\Omega_{m}=0.27, \Omega_{\Lambda}=0.73\right)$. The contribution from radiation is neglected for the SNIa fit, and the universe is assumed to be flat. The Hubble parameter is marginalized over in all of the fits (by maximizing the likelihood)

In Fig. 3 we show a contour plot of coefficients $(\mu, \beta)$ corresponding to $\Delta \chi^{2} \equiv \chi^{2}-\chi_{\Lambda \mathrm{CDM}}^{2}$, where we have fixed $\Omega_{m}=0.27, \Omega_{\mathrm{sc}}=0.01$. The region above the blue (short-dashed) curve shows where the $\chi^{2}$ is smaller than $\chi_{\Lambda \mathrm{CDM}}^{2}$, indicating a nominally better fit to the data. The green and red curves indicate contours where $\Delta \chi^{2}$ is -2 and -4 , respectively. The reduced $\chi^{2}$ for the $\Lambda$ CDM model is $201.6 /(194-1) \approx 1.045$, so an equal reduced $\chi^{2}$ is achieved with the model considered here (with two extra parameters), when $\Delta \chi^{2} \approx-2.1$.

The dependence on the coupled matter parameter $\Omega_{\mathrm{sc}}$ is very weak, whereas the uncoupled component plays a significant role. For example, with large values, $\Omega_{m} \geq$ 0.4 , the contours shift such that all of the considered $(\beta, \mu)$ parameter space gives a larger $\chi^{2}$ than the $\Lambda$ CDM value. Small values, $\Omega_{m} \leq 0.2$, lead to the same conclusion. In this section, our main purpose is to demonstrate the

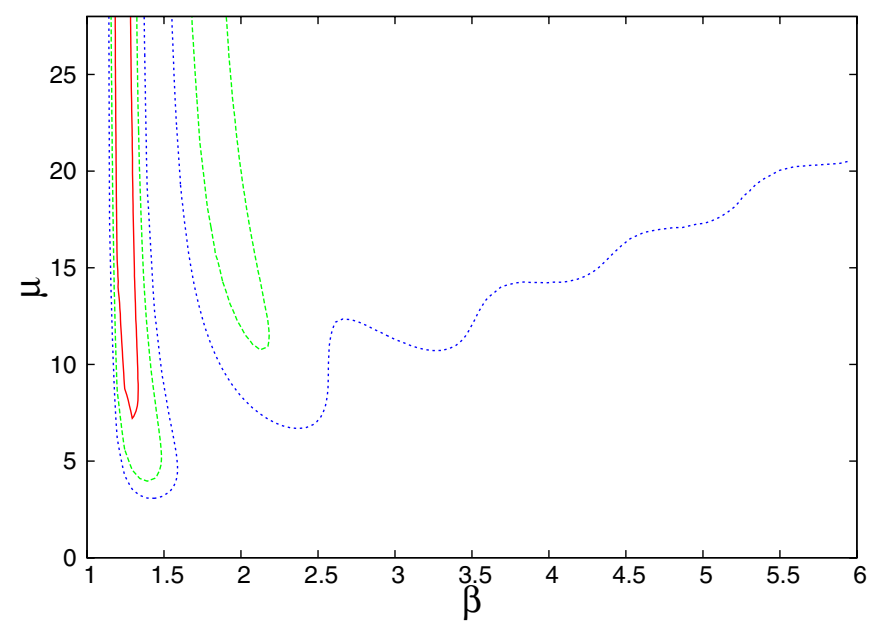

FIG. 3 (color online). Contour plot of $\Delta \chi^{2}\left(\Omega_{\tilde{m}}=0.27, \Omega_{m}=\right.$ 0.01). The red (solid) contour corresponds to -4 , the green (long-dashed) contour to -2 , and the blue (short-dashed) contour to 0 . 


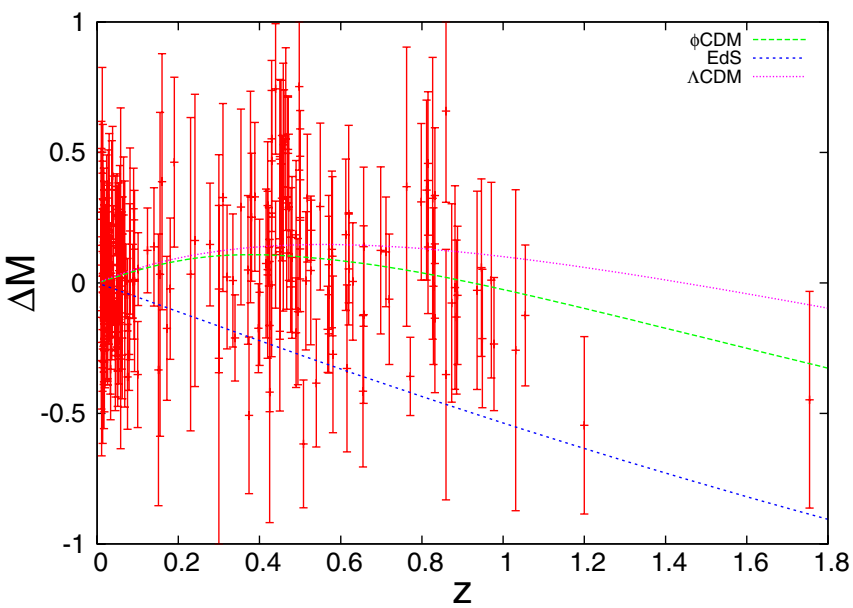

FIG. 4 (color online). Luminosity vs distance with respect to the empty universe for $(\beta=1.3, \mu=8)$ (green long-dashed line), the $\Lambda \mathrm{CDM}$ model (purple dot-dashed line) and EdS (blue short-dashed line) along with the SNIa data.

properties of the model considered here and to show how it can fit the current SNIa data very well. A more complete parameter scanning is left for future study.

In order to understand qualitatively why $\Delta \chi^{2}$ is negative for a range of parameter values, we show the luminosity distance plot as a function of redshift with respect to the empty universe, $\Delta M$, for a set of parameters $(\beta=1.3$, $\mu=8$ ), for which $\chi^{2}-\chi_{\Lambda \mathrm{CDM}}^{2}=-4.2$, in Fig. 4. From the figure, we see how for these parameters the luminosity distance at low redshifts closely follows that of the $\Lambda \mathrm{CDM}$ but, at later times, curves down more quickly, fitting the high redshift data points better.

\section{STRINGY PHYSICS AND COSMIC ACCELERATION}

Although we have seen that stringy SCDM can pave the way for a late-time acceleration phase, challenges remain to connect string theory with our coupled quintessence model in a quantitative way. First, our numerical analysis involving supernovae data suggests that we require $\mu / \beta \geq$ 2 and $\beta \geq 1.4$ for a good fit. The parameters are further pushed up if one also considers bounds coming from BBN: During the radiation era $\left(\mu_{r}=0\right)$ we have

$$
\tilde{\rho}_{\phi}=\frac{5}{6\left(\beta^{2}-1\right)} \tilde{\rho}_{r}
$$

BBN considerations provide an upper bound on the dark energy abundance, $\Omega_{\phi}<0.21$ during the radiation era. From Eq. (52) we see that for this to be satisfied one needs $\beta>2$.

Second, it is clear that the success of our cosmological scenario relies on having a very small abundance of CDM and SCDM in the early universe, and it would not be much progress if we simply shift the usual problem of quintes- sence models, namely, the problem of having to invoke a new and extremely small mass hierarchy (namely, the $\mathrm{eV}$ mass scale), to a problem of an unnaturally small initial abundance of the SCDM particles. Thus, in order to truly address the cosmic acceleration issue one needs to explain quantitatively why we expect such small abundances. In this section we address both of these issues, starting with the first.

\section{A. Quantum stringy corrections}

One may argue that we can obtain the required values of $\mu, \beta$ if stringy SCDM is coupled to several scalar fields [49]. This is because, depending upon which linear combinations are stabilized, the effective $\mu$ for $\phi$ (the linear combination which is rolling) can range from 0 to $\sqrt{\sum_{I} \mu_{I}^{2}}$, where $\mu_{I}$ 's are the exponents of the couplings to the different scalar fields, $\phi_{I}$ 's. Analogous arguments hold for $\beta$. However, rather than invoking many scalar fields, here we furnish an example which shows how quantum corrections involving stringy loops may be able to provide us with a significantly large value of $\mu$.

In the string frame, quantum string loop corrections will, in general, modify the couplings of the dilaton to the different fields:

$$
S=\int d^{10} x\left[C(\phi) R+K(\phi)(\partial \phi)^{2}-V(\phi)\right]
$$

where the functions $C(\phi), K(\phi)$, and $V(\phi)$ are expected to have a Taylor series expansion in terms of the inverse string coupling constant $g_{s}^{-1} \equiv e^{-\phi}[6]$,

$$
C(\phi)=\sum_{n=0}^{\infty} C_{n} e^{-n \phi},
$$

and similarly for $K(\phi)$ and $V(\phi)$. We recover the classical action (1) when all coefficients, except $C_{2}=1$ and $K_{2}=$ 4 , vanish. Now, let us suppose that the first nonzero coefficients of $C(\phi)$ and $K(\phi)$ occur at the nth level, while $V_{0}=0$ [53]. Then, as $\phi \rightarrow \infty$, the action is given by

$$
S \approx \int d^{10} x\left[e^{-n \phi} R+e^{-n \phi} K_{n}(\partial \phi)^{2}-V_{1} e^{-\phi}\right] .
$$

To go to the Einstein frame, we perform a conformal transformation,

$$
\hat{g}_{\hat{m} \hat{n}} \rightarrow e^{-2 n \phi /(\stackrel{D}{D}+2)^{g} \hat{g}_{\hat{m} \hat{n}},}
$$

followed by rescalings,

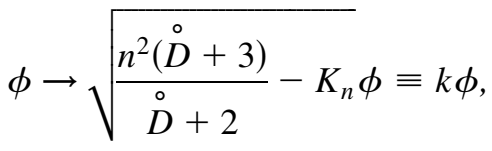

to make the kinetic terms for the dilaton canonical. After dimensional reduction to four dimensions, the action reads 


$$
S=\frac{1}{16 \pi G} \int d x^{4} \sqrt{-g}\left[R-\partial_{m} \phi \partial^{m} \phi-V_{1} e^{-2 \beta \phi}\right],
$$

where

$$
2 \beta=\frac{\stackrel{\circ}{D}+2-2 n}{k(\stackrel{\circ}{D}+2)} .
$$

Next, we look at the DBI action for $\stackrel{\circ}{D}$-branes and perform the conformal rescalings (2) to obtain the effective four-dimensional action for the gas of 6-branes:

$$
S_{\text {brane }}=\int d^{4} x \sqrt{-g} \tilde{\rho}=\int d^{4} x \sqrt{-g} \rho_{0} e^{-2 \mu_{\text {sc }} \phi}\left(\frac{a}{a_{0}}\right)^{-3},
$$

with

$$
\mu_{\mathrm{sc}}=\frac{\stackrel{\circ}{D}(n-1)+n-2}{k(\stackrel{\circ}{D}+2)} .
$$

Note that we want $\mu / \beta$ to be positive. Substituting $\stackrel{\circ}{D}=6$, one finds that this is possible only for $n=2,3,4$ :

$$
\begin{gathered}
\text { for } n=2, \quad \frac{\mu_{\mathrm{sc}}}{\beta}=1, \\
\text { for } n=3, \quad \frac{\mu_{\mathrm{sc}}}{\beta}=\frac{13}{4} \approx 3.13, \\
\text { for } n=4, \quad \frac{\mu_{\mathrm{sc}}}{\beta}=10 .
\end{gathered}
$$

From Eq. (57) it is also clear that, depending on the value of $K_{n}, k$ could be small, hence making $\mu_{\mathrm{sc}}, \beta$ large.

\section{B. Primordial inflation and the scale of current acceleration}

It is clear that a resolution of the dark energy problem should not only involve (a) a tracking mechanism which explains why the dark energy density has always been close to the matter density, and not just today (and this is most certainly the case in our model), but also (b) a parameter (since we do not want to introduce a hierarchical $\mathrm{eV}$ mass scale) which governs when the dark energy comes out of the tracking phase and starts to dominate the universe. Moreover, this parameter should be such that its expected value can naturally explain why we are entering this phase of acceleration so late in the day.

Now, in our model, acceleration commences approximately when SCDM becomes comparable to ordinary CDM. Since SCDM redshifts more slowly than CDM, this means that in the early universe (say just after inflation) the energy density in SCDM was much smaller than that of CDM. This could happen, for example, if SCDM is produced very scantily during the reheating process, as argued in Sec. II. More importantly, one realizes that SCDM candidates are stringy winding states in nature. One expects these winding states to be present in the very early preinflationary universe with approximately string scale energy densities. However, as our universe expands exponentially during inflation, this gas of branes is going to become very dilute. Since the slope of the potential decreases exponentially as $\phi$ increases, we expect the dilaton $\phi$ to be effectively frozen during the phase of inflation. As a result, the SCDM energy density will redshift as $a^{-3}$.

Thus, at the end of inflation, while the energy density in ordinary matter would be given by the reheat temperature, the energy density in the primordial gas of SCDMs would be much smaller due to inflationary dilution. In fact, the larger the number of $e$-foldings, $\mathcal{N}$, the greater the dilution, and therefore the longer it will take for SCDM to catch up with CDM, and the smaller the scale $\left(\equiv M_{A}\right)$ would be at which the second phase of acceleration commences. If $M_{A} \equiv M_{p} 10^{-A}$, then one finds (see the Appendix for details)

$$
A=\frac{R\left(1+4 \mu / \beta-3 \omega_{s c}\right)+(1.3 \mathcal{N}-27)\left(1+\omega_{\mathrm{sc}}\right)}{4\left(\mu / \beta-\omega_{\mathrm{sc}}\right)}
$$

where $M_{P} 10^{-R}$ corresponds to the reheat temperature.

We are now in a position to estimate the scale at which the second acceleration phase starts. As a first example, let us choose $\omega_{\mathrm{sc}}=0$, and $\mu / \beta=1 / 2$, the minimal value required for acceleration and also approximately what one obtains for the 6-branes. For the minimal number of $e$-foldings, $\mathcal{N}=60$, and assuming reheating to GUTscale temperatures $M_{\text {reh }}$, i.e. $M_{\text {reh }} \sim 10^{-3} M_{p}$ or $R=3$, one finds from (62) that $M_{A} \sim 10^{-30} M_{p}=10^{-2} \mathrm{eV}$. We remind the reader that the current energy density corresponds to the energy scale $10^{-3} \mathrm{eV}$. For $\omega_{\text {sc }}=1 / 3$, i.e. radiative SCDM, one needs $\mu / \beta=1$ for acceleration. Then, using previous values for $\mathcal{N}$ and $R$, we again find $M_{A} \sim 10^{-30} M_{p}$.

The agreement, however, is not as dramatic as it seems. The more detailed numerical analysis performed with $\omega_{\mathrm{sc}}=0$ revealed that we get good agreement with the supernova data for values of $\mu / \beta \geq 2$. Demanding $A \sim$ 31 and $\mu / \beta \sim 2$, one "predicts" the number of primordial inflation to be $\mathcal{N} \sim 200$ if one demands that the second stage of acceleration is beginning at the present time. Estimates with $\omega_{\mathrm{sc}}=1 / 3$ and $\mu / \beta \sim 5$ (49) yield a similar estimate for $\mathcal{N}$, namely $\mathcal{N} \sim 250$. Of course, this number depends on the reheat temperature, and on the precise preinflationary initial energy density of SCDM, but these dependences are relatively weak as can be seen from (62). Thus, (62) should be treated as a relation between $A$ and $\mathcal{N}$. Moreover, since $A=A(\mathcal{N})$ is a linear relationship, there is no fine-tuning involved; a small 
variation in $\mathcal{N}$ results in only a small variation in $A$. This is, of course, to be contrasted with the fine-tuning problem in $\Lambda \mathrm{CDM}$ models where the difference of two large numbers is supposed to yield a very small cosmological constant. Hence, a small variation in one of the large numbers causes a huge variation in $\Lambda$.

\section{CONCLUSIONS}

In this paper we have introduced the concept of "stringy cold dark matter" (SCDM). From the point of view of our four-dimensional space-time, SCDM are particles which couple nontrivially [with a coupling $\exp (2 \mu \phi)$ ] to the dilaton. Candidates for SCDM from string theory are branes wrapping some or all of the compact spatial dimensions. We have studied the cosmology of these modes in the context of a type IIA supergravity action to which we have added a runaway potential $V_{0} \exp (-2 \beta \phi)$ for the dilaton $\phi$ (as explained in the text, such potentials are generally believed to be generated when going beyond the classical description). We have shown the existence of cosmological tracking solutions in the context of which the dilaton becomes a candidate for dark energy [54].

Our model contains radiation, regular dark matter (which couples very weakly if at all to the dilaton), SCDM, and the dilaton. Assuming that our fourdimensional space-time underwent a period of primordial inflation, the initial energy density in SCDM is expected to be exponentially suppressed compared to the density of regular CDM and radiation (which can be produced during reheating after inflation). In this context, the universe is initially dominated by radiation, and we show that the energy density in the dilaton tracks that of radiation until the dilaton gets stuck at the minimum of its effective potential, a potential formed from the dilaton potential (potential with a negative slope) and the terms with positive slope coming from the interactions of the dilaton with SCDM. From then on, the density in SCDM and in the dilaton redshifts more slowly than that of ordinary matter. During this phase, the density in the dilaton is greater than that in SCDM. Once the dilaton energy begins to dominate, a period of acceleration begins, provided $\mu / \beta$ is sufficiently large.

Compared to models of quintessence, an advantage of our approach is that it does not require the introduction of a new mass hierarchy (a new mass scale of about $1 \mathrm{eV}$ ). In order to explain why the late-time acceleration begins at the present time, a sufficient suppression of the number density of SCDM states is required. Such a suppression is naturally generated by a period of cosmological inflation. In fact, the number $\mathcal{N}$ of $e$-foldings of inflation required to suppress the SCDM density is (for GUT-scale reheating) larger but of the same order of magnitude as the minimal value of $\mathcal{N}$ required for successful inflation. Thus, in our framework the "coincidence mystery" of why dark energy is beginning to dominate today is tied to the duration of the period of inflation.

For our scenario to work, a sufficiently large value of $\mu / \beta$ is required. Even larger values of this ratio are required to be consistent with big bang nucleosynthesis. At the level of the classical action, it is not possible to obtain such large couplings between the SCDM particle and single scalar moduli. The cumulative effect of coupling the SCDM particle to several different scalar fields may help resolve this difficulty, as may nonperturbative effects. Further research on this issue is required. A proper understanding of $\alpha^{\prime}$ and string loop corrections is also necessary in order to study the stability of this model; we have assumed the validity of Ref. [9] in the low energy regime we are interested in. It is an important task to have known these corrections to all orders; however, this is not the main focus of this paper.

It would be interesting to study further consequences of the existence of SCDM in the present universe, following the approaches of [56] to study cosmological consequences and of [57] to study astrophysical and particle physics aspects.

\section{ACKNOWLEDGMENTS}

T. B. is supported by the NSERC Grant No. 204540. The work of R. B. is supported by an NSERC Discovery grant and by the Canada Research Chair Program.

\section{APPENDIX}

We start by assuming that, in the very early universe, the energy density of the stringy SCDM and of $\phi$ is given by the string scale, $\tilde{\rho}_{\mathrm{sc}} \sim V(Q) \sim M_{\mathrm{str}}^{4}$. Now, a small hierarchy between the string scale and the scale of inflation ensures that $Q$ or SCDM play no role in the inflationary mechanism and that $\phi$ is effectively frozen during this phase. As a result, the SCDM energy density redshifts as $a^{-3\left(1+\omega_{\mathrm{sc}}\right)}$ with the rapid expansion of our universe. Accordingly, after the end of inflation the SCDM energy density will be given by

$$
\tilde{\rho}_{\mathrm{sc}}\left(t_{R}\right)=\rho_{I} e^{-3\left(1+\omega_{\mathrm{sc}}\right)} \mathcal{N}
$$

where $\mathcal{N}$ is the number of $e$-foldings of inflation, $t_{R}$ stands for the time of the end of inflation (the time of reheating), and $\rho_{I}$ is the energy density at the beginning of inflation (when the inflaton potential becomes dominant). It is a good assumption that the SCDM density will not be suppressed relative to that of other matter between the initial time and the onset of inflation.

We choose, by convention, $\phi\left(t_{R}\right)=0$ and thus $V(\phi)=$ $M_{\mathrm{str}}^{4} e^{-2 \beta \phi}$, or $V_{0}=M_{\mathrm{str}}^{4}$. Let us also assume, just for the purpose of illustration, that reheating is very fast, i.e. that the period of inflation is immediately followed by a radiation period (this is likely to be the case if reheating is driven by parametric resonance). In this case, after reheating, the 
radiation energy density is given by

$$
\tilde{\rho}_{r}\left(t_{R}\right)=\rho_{I} \equiv M_{p}^{4} 10^{-4 R},
$$

where $R$ sets the scale of inflation (which is $10^{-R} M_{p}$ ). It is now clear from (A1) and (A2) that after the end of inflation for $\mathcal{N} \geq 60$, and $\omega_{\text {sc }} \geq 0$, the radiation energy density is a lot larger than the SCDM density. This is essentially why it takes a long time for $\tilde{\rho}_{\mathrm{sc}}$ to catch up with radiation density. As we have seen, once it does, a second phase of accelerated expansion begins. Let us therefore try to estimate when SCDM becomes comparable to ordinary matter/ radiation.

After inflation, all the scalar fields are free to roll. The energy density of the $\phi$ field will first track the radiation and then matter energy density, maintaining a constant ratio with them. Thus, in the radiation era

$$
e^{-2 \beta \phi} \sim a^{-4} \Rightarrow e^{2 \mu \phi} \sim a^{4 \mu / \beta} .
$$

Hence the radiation and SCDM densities redshift differently,

$$
\begin{aligned}
\tilde{\rho}_{\mathrm{sc}} & =\rho_{I} e^{-3\left(1+\omega_{\mathrm{sc}}\right)} \mathcal{N}\left(\frac{a}{a_{E}}\right)^{4 \mu / \beta-3\left(1+\omega_{\mathrm{sc}}\right)} \\
& =M_{P}^{4} 10^{-1.3\left(1+\omega_{\mathrm{sc}}\right)} \mathcal{N}-4 R\left(\frac{a}{a_{E}}\right)^{4 \mu / \beta-3\left(1+\omega_{\mathrm{sc}}\right)}
\end{aligned}
$$

and

$$
\tilde{\rho}_{r}=M_{p}^{4} 10^{-4 R}\left(\frac{a}{a_{E}}\right)^{-4}
$$

After the radiation-matter equality, when the energy density of the universe has fallen to about $10^{-108} M_{p}^{4}, \phi$ starts to track the matter density instead,

$$
e^{-2 \beta \phi} \sim a^{-3} \Rightarrow e^{2 \mu \phi} \sim a^{3 \mu / \beta} .
$$

Now, one can obtain the ratio $a_{\mathrm{eq}} / a_{R}$, where " $t_{\mathrm{eq}}$ " is the time of radiation-matter equality and $a_{\mathrm{eq}}$ is the value of the scale factor at that time, and then substitute it in (A4) to obtain the energy density of SCDM at the equality epoch:

$$
\tilde{\rho}_{\mathrm{sc}}\left(\left(t_{\mathrm{eq}}\right)=M_{p}^{4} 10^{-1.3\left(1+\omega_{\mathrm{sc}}\right) \mathcal{N}-4 R} 10^{(27-R)\left(4 \mu / \beta-3\left(1+\omega_{\mathrm{sc}}\right)\right)} .\right.
$$

The evolution of strongly coupled and ordinary matter is then given by

$$
\tilde{\rho}_{\mathrm{sc}}=\tilde{\rho}_{\mathrm{sc}}\left(t_{\mathrm{eq}}\right)\left(\frac{a}{a_{\mathrm{eq}}}\right)^{3 \mu / \beta-3\left(1+\omega_{\mathrm{sc}}\right)}
$$

and

$$
\tilde{\rho}_{m}=M_{p}^{4} 10^{-108}\left(\frac{a}{a_{\mathrm{eq}}}\right)^{-3}
$$

respectively.

Now, as explained before, $\tilde{\rho}_{\text {sc }} \sim \tilde{\rho}_{m} \equiv M_{A}^{4}$ corresponds to the acceleration epoch. If $M_{A} \equiv M_{p} 10^{-A}$, then from (A8) and (A9) we have

$$
A=\frac{R\left(1+4 \mu / \beta-3 \omega_{s c}\right)+(1.3 \mathcal{N}-27)\left(1+\omega_{\mathrm{sc}}\right)}{4\left(\mu / \beta-\omega_{\mathrm{sc}}\right)} .
$$

(A10)
[1] D. N. Spergel et al. (WMAP Collaboration), Astrophys. J. Suppl. Ser. 148, 175 (2003).

[2] F. Quevedo, Prepared for ICTP Spring School on Superstrings and Related Matters, Trieste, Italy, 2002.

[3] The coherent oscillations of the Peccei-Quinn axion can explain the origin of CDM within a minimal extension of the SM (see e.g. Ref. [4] for a review) which provides a dynamical mechanism for obtaining a small $\theta_{\mathrm{QCD}}$ and thus solves the strong CP problem.

[4] E. W. Kolb and M.S. Turner, The Early Universe (Addison-Wesley, Redwood City, 1990).

[5] S. Perlmutter et al. (Supernova Cosmology Project Collaboration), Astrophys. J. 517, 565 (1999); A. G. Riess et al. (Supernova Search Team Collaboration), Astron. J. 116, 1009 (1998); Astrophys. J. 607, 665 (2004).

[6] M. Gasperini, F. Piazza, and G. Veneziano, Phys. Rev. D 65, 023508 (2002).

[7] T. R. Taylor, Phys. Lett. B 252, 59 (1990).

[8] P. Binetruy, M. K. Gaillard, and Y. Y. Wu, Nucl. Phys. B481, 109 (1996); G. R. Dvali and Z. Kakushadze, Phys.
Lett. B 417, 50 (1998); S. A. Abel and G. Servant, Nucl. Phys. B597, 3 (2001); E. Silverstein, hep-th/0405068.

[9] T. Damour and A. M. Polyakov, Nucl. Phys. B423, 532 (1994).

[10] In the strong coupling regime, the gauge couplings can be determined by algebraic quantities, such as the rank and Casimir invariants of the gauge group [6].

[11] C. Wetterich, Nucl. Phys. B302, 668 (1988).

[12] B. Ratra and P. J.E. Peebles, Phys. Rev. D 37, 3406 (1988).

[13] R. R. Caldwell, R. Dave, and P. J. Steinhardt, Phys. Rev. Lett. 80, 1582 (1998).

[14] P. Jaikumar and A. Mazumdar, Phys. Rev. Lett. 90, 191301 (2003).

[15] R. H. Brandenberger and C. Vafa, Nucl. Phys. B316, 391 (1989).

[16] S. Alexander, R. H. Brandenberger, and D. Easson, Phys. Rev. D 62, 103509 (2000).

[17] We are interested in the late-time cosmology of string gas, unlike the dynamics of the stringy epoch close to the selfdual radius, where the interaction rates are determined by 
taking into account the stringy corrections [18].

[18] R. Danos, A. R. Frey, and A. Mazumdar, Phys. Rev. D 70, 106010 (2004).

[19] S. B. Giddings, S. Kachru, and J. Polchinski, Phys. Rev. D 66, 106006 (2002).

[20] S. Kachru, R. Kallosh, A. Linde, and S. P. Trivedi, Phys. Rev. D 68, 046005 (2003).

[21] S. Watson, Phys. Rev. D 70, 066005 (2004).

[22] S. P. Patil and R. Brandenberger, Phys. Rev. D 71, 103522 (2005).

[23] S. P. Patil and R. H. Brandenberger, J. Cosmol. Astropart. Phys. 01 (2006) 005.

[24] R. Brandenberger, Y. K. Cheung, and S. Watson, J. High Energy Phys. 05 (2006) 025.

[25] S. Watson and R. Brandenberger, J. Cosmol. Astropart. Phys. 11 (2003) 008.

[26] T. Biswas and A. Mazumdar, hep-th/0408026.

[27] T. Biswas, R. Brandenberger, D. A. Easson, and A. Mazumdar, Phys. Rev. D 71, 083514 (2005).

[28] A. Berndsen, T. Biswas, and J. M. Cline, J. Cosmol. Astropart. Phys. 08 (2005) 012.

[29] In the above, $a(t)$ is the scale factor and $a_{0}$ is its value at some reference time at which the energy density is given by $\rho_{0}$.

[30] The reason one cannot straightforwardly substitute $p=1$ in (12) to get the exponent for winding strings lies in the fact that the fundamental string action does not contain any dilaton coupling, unlike the DBI action for the solitonic branes which does.

[31] T. Battefeld and S. Watson, J. Cosmol. Astropart. Phys. 06 (2004) 001.

[32] L. F. Abbott, E. Farhi, and M. B. Wise, Phys. Lett. 117B, 29 (1982).

[33] A. D. Dolgov and A.D. Linde, Phys. Lett. 116B, 329 (1982).

[34] J. H. Traschen and R. H. Brandenberger, Phys. Rev. D 42, 2491 (1990).

[35] L. Kofman, A. D. Linde, and A. A. Starobinsky, Phys. Rev. Lett. 73, 3195 (1994).

[36] We assume instantaneous thermalization, which need not be a correct assumption; for details see [37].

[37] R. Allahverdi and A. Mazumdar, hep-ph/0505050; P. Jaikumar and A. Mazumdar, Nucl. Phys. B683, 264 (2004).

[38] D. J. H. Chung, E. W. Kolb, and A. Riotto, Phys. Rev. D 59, 023501 (1999).
[39] S. S. Gubser and P. J. E. Peebles, Phys. Rev. D 70, 123510 (2004).

[40] M.S. Bremer, M. J. Duff, H. Lu, C. N. Pope, and K. S. Stelle, Nucl. Phys. B543, 321 (1999); T. Biswas and P. Jaikumar, J. High Energy Phys. 08 (2004) 053; Int. J. Mod. Phys. A 19, 5443 (2004).

[41] S. M. Carroll, Phys. Rev. Lett. 81, 3067 (1998).

[42] Other mechanisms such as the "least coupling principle" [9] or the chameleon mechanism [43] have also been proposed to explain the small couplings, but these mechanisms seem unlikely to be compatible with our model.

[43] J. Khoury and A. Weltman, Phys. Rev. Lett. 93, 171104 (2004); D. F. Mota and J. D. Barrow, Phys. Lett. B 581, 141 (2004); Mon. Not. R. Astron. Soc. 349, 291 (2004); J. Khoury and A. Weltman, Phys. Rev. D 69, 044026 (2004).

[44] G. Huey, P. J. Steinhardt, B. A. Ovrut, and D. Waldram, Phys. Lett. B 476, 379 (2000).

[45] In the above, we have made use of the adiabatic approximation (36).

[46] The exact sequence of these two events is actually not important for the basic mechanism to work. However, for the relevant values of $\mu / \beta$ the two events occur in the order described.

[47] S. Sarkar, astro-ph/0205116.

[48] B. J. Barris et al., Astrophys. J. 602, 571 (2004).

[49] Note that invoking several scalar fields has also been shown to make it easier to realize a period of cosmological inflation [50-52].

[50] A. R. Liddle, A. Mazumdar, and F. E. Schunck, Phys. Rev. D 58, 061301 (1998); E. J. Copeland, A. Mazumdar, and N. J. Nunes, Phys. Rev. D 60, 083506 (1999).

[51] R. Brandenberger, P. M. Ho, and H.c. Kao, J. Cosmol. Astropart. Phys. 11 (2004) 011.

[52] A. Jokinen and A. Mazumdar, Phys. Lett. B 597, 222 (2004).

[53] One could consider more general conditions, but since this is only for illustration, we choose a simple ansatz.

[54] Cosmological tracking solutions from exponential potentials have also been considered in [55] and references therein.

[55] E. J. Copeland, A. R. Liddle, and D. Wands, Phys. Rev. D 57, 4686 (1998).

[56] A. Nusser, S. S. Gubser, and P. J. E. Peebles, Phys. Rev. D 71, 083505 (2005).

[57] G. Shiu and L. T. Wang, Phys. Rev. D 69, 126007 (2004). 